\title{
TELEMONITORAMENTO EM ENFERMAGEM PARA CLIENTES EM SITUAÇÃO DE ESTOMATERAPIA: EXPERIÊNCIA INOVADORA PARA O PROCESSO ENSINO-APRENDIZAGEM
}

TELEMONITORING IN NURSING FOR CLIENTS IN A STOMATHERAPY SITUATION: AN INNOVATIVE EXPERIENCE FOR THE TEACHING-LEARNING PROCESS

\section{Autores}

Beatriz Oliveira Nascimento. Acadêmica do 50 período da ENF/UERJ. Bolsista de extensão do projeto intitulado: Telemonitoramento em enfermagem para clientes em situação de estomaterapia: feridas, estomias e incontinência. Faculdade de Enfermagem da Universidade do Estado do Rio de Janeiro (Uerj). Rio de Janeiro. Brasil.

Email: beatriz.oliveitra16@gmail.com

Norma Valéria Dantas de Oliveira Souza Enfermeira. Doutora em Enfermagem. Especialista em Estomaterapia TISOBEST. Coordenadora da Clínica de Enfermagem em Estomaterapia da PPC/UERJ. Faculdade de Enfermagem da Universidade do Estado do Rio de Janeiro (Uerj). Rio de Janeiro. Brasil.

Email:norval_souza@yahoo.com.br

Déborah Machado dos Santos. Enfermeira. Doutora em Enfermagem. Especialista em Estomaterapia TISOBEST. Coordenadora da Clínica de Enfermagem em Estomaterapia da PPC/UERJ. Faculdade de Enfermagem da Universidade do Estado do Rio de Janeiro (Uerj). Rio de Janeiro. Brasil.

Email: debuerj@yahoo.com.br

Patrícia Alves dos Santos Silva. Enfermeira. Mestre em Enfermagem. Especialista em Estomaterapia TISOBEST. Enfermeira da Clínica de Enfermagem em Estomaterapia da PPC/UERJ. Faculdade de Enfermagem da Universidade do Estado do Rio de Janeiro (Uerj). Rio de Janeiro. Brasil.

Email: papatty@gmail.com

Recebido em: 24/01/2019 Aprovado em: 31/01/2019

DOl: 10.12957/interag.2018.39668

Relato

Resumo

Trata-se de relato de experiência sobre a participação em um projeto de extensão da Faculdade de Enfermagem intitulado "Telemonitoramento em Enfermagem para Clientes em Situação de Estomaterapia: feridas, estomias e incontinência". Os objetivos deste relato são: descrever a vivência no referido projeto, no recorte tem-

\section{Abstract}

This is a report of experience on participation in an extension project of the nursing faculty entitled "Telemonitoramento in Nursing for clients in a situation of stomatherapy: wounds, Stomas and incontinence". The objectives of this report are: to describe the experience in the aforementioned project, in the temporal period 
poral de abril a outubro de 2018; e discutir as contribuições das atividades extensionistas para o ensino e para a assistência de enfermagem. O projeto é desenvolvido na Policlínica Piquet Carneiro, especificamente na Clínica de Enfermagem em Estomaterapia Benedita Deusdará Rodrigues, cujo público- alvo são pessoas com feridas, estomas e incontinências, anal e urinário. Utiliza-se contato telefônico para fornecer e reforçar as orientações de enfermagem aos usuários do serviço com o fito dos mesmos desenvolver o autocuidado no domicílio. Estas orientações são norteadas por protocolos e registradas em formulário, os quais foram elaborados pela bolsista e pela orientadora do projeto, bem como pela coordenadora e equipe da referida clínica. Os resultados decorrentes das atividades extensionistas têm sido profícuos para a assistência, pois contribuem para prevenir complicações e melhorar a qualidade de vida dos usuários, revelando-se também como um campo diferenciado para o processo ensino-aprendizagem da bolsista.

Palavras- chave: Enfermagem;

Telemonitoramento; Processo educativo; Estomaterapia

Área Temática: Saúde

Linha Temática: Tecnologia da informação from April to October 2018; and discuss the contributions of Extensionist activities for teaching and nursing care. The project is developed in Policlínica Piquet Carneiro, specifically in the nursing clinic in Stomatherapy Benedita Deusdará Rodrigues, whose target audience are people with wounds, Stommas and incontinences, both in the urinary and the urine. Telephone contact is used to provide and reinforce nursing orientations to users of the service with the aim of developing self-care at home. These guidelines are guided by protocols and registered in form, which were elaborated by the scholarship holder and the project advisor, as well as by the Coordinator and staff of the aforementioned clinic. The results resulting from the extensionist activities have been fruitful for care, as it contributes to prevent complications and improve the quality of life of users, also revealing itself as a differentiated field for the process Teaching-learning of the scholar.

Keywords: Nursing; Telemonitoring; Educational process; Stomatherapy

\section{Introdução}

Este manuscrito tem como foco relatar a experiência vivida em um projeto de extensão intitulado "Telemonitoramento em Enfermagem para Clientes em Situação de Estomaterapia: feridas, estomias e incontinência". Nesta perspectiva, apontam-se os seguintes objetivos: descrever a vivência no referido projeto, no recorte temporal de abril a outubro de 2018; e discutir as contribuições das atividades extensionistas para o ensino e para a assistência de enfermagem.

A Estomaterapia é uma especialidade da enfermagem que visa cuidar de pessoas com lesões de pele, estomias e incontinências, anal e urinária, as quais são alterações de saúde que apresentam crescente e elevada incidência e prevalência. ${ }^{1}$ 
Neste sentido, observa-se um quantitativo preocupante de pessoas tanto com feridas agudas, na maior parte de etiologias acidentais, quanto crônicas, originadas por patologias como hanseníase, leishmaniose, AIDS, lúpus eritematoso, doença vascular periférica, diabetes melitus, câncer, entre outras. ${ }^{2}$

Especialmente no caso do câncer, o problema de saúde pública agrava-se, pois, o crescimento dos casos de neoplasia colorretal nos últimos dez anos, segundo dados do Ministério da Saúde, tem resultado no aumento assustador de pessoas com estomias. ${ }^{3}$

Além disto, há de se citar os casos "silenciosos", mas assustadores em termos de ocorrência e de baixa da qualidade de vida de pessoas com incontinências, urinária e anal. Especialmente as mulheres sofrem silenciosamente com diversos tipos de incontinência urinária, pois essa população esconde, por vergonha, essa patologia. Outrossim, aponta-se a incidência significativa de homens com incontinência urinária decorrente de sequela cirúrgica da prostatectomia radical para cura e/ou tratamento do câncer de próstata. ${ }^{4}$

Tais problemáticas de saúde geram repercussões para as dimensões física, psíquica e social dos indivíduos. Destaca-se, por exemplo, alteração da imagem corporal e da autoestima; afastamentos das atividades laborais, que geram consequências econômicas para a pessoa e sua família; complicações fisiológicas que resultam em elevada morbimortalidade. Portanto, faz-se mister atuações e estratégias diversificadas e inovadoras dos profissionais com o fito de contribuir com a melhor qualidade de vida e prevenção de agravos à saúde destas pessoas. ${ }^{1}$

No contexto da Estomaterapia, uma estratégia relevante para evitar complicações e garantir bem-estar aos indivíduos, é a orientação para o autocuidado e para a adesão ao tratamento. Nesta perspectiva, há várias formas para desenvolver esse processo de orientação, dentre elas destacam-se o uso do computador para consultas virtuais, a utilização do acompanhamento por telefone, vídeo conferência e mensagens de celular. ${ }^{5}$

A utilização destas tecnologias no trabalho em saúde e enfermagem propiciou a criação de inovadoras maneiras de cuidar e assistir, que podem ser denominadas de: telessaúde, telemedicina, a teleassistência, a tele-enfermagem e o telemonitoramento em enfermagem. ${ }^{6}$

O telemonitoramento em enfermagem caracteriza-se pelo uso das telecomunicações e das tecnologias computacionais com o objetivo de prestar cuidados de enfermagem. É a enfermagem à distância, mediada no todo ou em parte, como uma importante estratégia para direcionar a assistência. ${ }^{6}$

A utilização do telefone nos serviços de saúde possibilita ao paciente e a sua família rápido acesso ao profissional de saúde, diminuição do tempo de espera para a consulta, redução de tempo e custo na locomoção dos pacientes ao serviço, aumento na frequência dos contatos e do retorno do paciente, aumento do sentimento de segurança e apoio, diminuição de complicações de saúde e diminuição no tempo de recuperação dos problemas de saúde. ${ }^{6}$

Com base neste conhecimento, considerou-se relevante desenvolver o mencionado projeto de extensão, que tem como objetivo geral desenvolver o telemonitoramento, por meio do telefone, para clientes com lesão de pele de natureza diversa, estomias e incontinência urinária e anal.

A experiência neste projeto tem sido profícua para os usuários do serviço, para a equipe de enfermagem e, em especial, para a bolsista do projeto, a qual vislumbrou um contexto diferenciado para desenvolver o cuidado de enfermagem, incentivado o aprofundamento do conhecimento sobre Estomaterapia, telemonitoramento, comunicação e educação em saúde. 
Nesse sentido, visando compartilhar esta produtiva vivência, considerou-se relevante elaborar o presente manuscrito.

\section{Metodologia do trabalho no processo de Extensão}

O projeto em tela é desenvolvido na Clínica de Enfermagem em Estomaterapia Benedita Deusdará Rodrigues, situada na Policlínica Piquet Carneiro/UERJ. Tem-se como marco inicial da atuação da bolsista o mês de abril de 2018, quando houve as primeiras orientações e discussões sobre a metodologia do projeto e os objetivos que se pretendiam alcançar, os quais se caracterizam como:

1) Elaborar protocolos de orientação voltados para pessoas com comprometimento de pele, com estomias e com incontinência;

2) Produzir um formulário de registro de enfermagem para anotação acerca do processo de orientação efetuada pelo telemonitoramento enfermagem;

3) Realizar processo educativo, desenvolvido por meio do telemonitoramento, baseado em protocolos de orientação a pessoas com comprometimento de pele, estomias e incontinência;

4) Realizar registro de enfermagem acerca do processo educativo efetuado por meio de telemonitoramento;

5) Processar dados estatísticos acerca do atendimento por meio do telemonitoramento em enfermagem;

6) Desenvolver estudos científicos a partir da experiência vivenciada com o telemonitoramento;

7) Participar de eventos científicos a fim de apresentar os resultados dos estudos efetuados.

A fim de alcançar os objetivos descritos, houve reuniões periódicas entre a bolsista, a coordenadora do projeto, a coordenadora de enfermagem da clínica e a equipe que compõe o trabalho neste espaço de cuidar. Tais reuniões tinham como fito capacitar a bolsista sobre temas ligados à Estomaterapia; discutir o processo de trabalho na clínica; refletir sobre a relevância da atuação do enfermeiro estomaterapeuta para a qualidade de vida de pessoas com problemas de pele, estomas e incontinências; instrumentalizá-la para a elaboração dos protocolos, formulários e registros de enfermagem e dos dados estatísticos relativos aos atendimentos na clínica.

Além disso, houve o treinamento da bolsista a fim de desenvolver a habilidade de orientar ao telefone utilizando linguagem clara, objetiva e apropriada a especificidade cultural e educacional dos usuários. Outrossim, enfatiza-se o processo de capacitação efetuado para o adequado registro das orientações de enfermagem no prontuário dos usuários. Destaca-se também as horas de observação de campo efetuadas pela bolsista acerca dos atendimentos efetuados pelas enfermeiras como estratégia de apropriá-las em relação aos procedimentos de enfermagem, tornando concreto o que iria orientar e reforçar no telemonitoramento.

\section{A experiência de aprendizado no projeto}

Como resultado, destaca-se que ao longo de seis meses foram realizados 84 telemonitoramentos, sendo 19 para pacientes em situação de estomia, 20 para incontinência e 45 em situação de ferida.

Além disso, a atuação da bolsista contribuiu para a criação de três formulários, objetivando nortear o telemonitoramento de pessoa com ferida, estomia e incontinência. Tais 
formulários foram idealizados também para possibilitar o registro das alterações de saúde informadas, propiciando a avaliação de retorno imediato ou tardio ao serviço, assim como a identificação das dúvidas do paciente, esclarecendo e reforçando orientações fornecidas nas consultas presenciais.

Outro resultado relevante foi a confecção de um folder de orientação voltado para pacientes com incontinência. Este material instrutivo ilustra, por meio de figuras, como desenvolver os exercícios de fortalecimento do assoalho pélvico, bem como reforça orientações efetuadas presencialmente e a distância, abordando igualmente as complicações que as pessoas com incontinência podem desenvolver.

Salienta-se a contribuição na busca ativa dos usuários para obter informações sobre os motivos que levam os usuários a abandonar o tratamento, enfatizando-se a importância do retorno imediato às consultas, possibilitando o reagendamento.

Outrossim, registra-se a elaboração de uma ficha de controle do telemonitoramento para organização dos telefonemas atendidos e não atendidos, a fim de que haja o retorno no período adequado; motivos pelos quais ocorrem novos agendamentos e atualização do censo dos usuários atendidos na clínica.

Uma experiência vivida que deve ser salientada como relevante foi a natureza e a variedade das orientações efetuadas, as quais envolveram: a frequência de troca do equipamento coletor utilizado pelos usuários com estomias; a necessidade e maneira de realização dos exercícios de fortalecimento do assoalho pélvico; os alimentos a serem evitados pelas pessoas em situação de incontinência; a importância do acompanhamento nas consultas presenciais; o esclarecimento das dúvidas dos usuários/familiares; e a detecção precoce de complicações de saúde a partir da abordagem através do telefone.

Obteve-se também a oportunidade de apresentação do projeto em um evento científico, e a conquista de menção honrosa decorrente da elaboração de uma redação sobre direitos humanos e o contexto da Estomaterapia, experiência singular que deu concretude à relevância do projeto.

\section{Considerações finais}

Ser integrante deste projeto tem sido de grande aprendizado para a bolsista, pois possibilita crescer como acadêmica, contribuindo para o ensino na enfermagem, visto que são conhecimentos pouco trabalhados na graduação.

Constatou-se a possibilidade de formar um vínculo mais estreito com os usuários e familiares, além de oferecer um cuidado individualizado. Verificou-se que é possível explorar diferentes estratégias de aprendizagem, além de permitir uma interação com a equipe de enfermagem da presente clínica.

Este projeto abriu oportunidades para a bolsista se apresentar pela primeira vez em um evento científico, o que despertou grande interesse em compartilhar ainda mais componentes deste processo para que estudantes, profissionais e outros indivíduos possam tomar conhecimento desta área tão importante.

Em pouco tempo foi possível saber na prática que na enfermagem existem diversos caminhos para ofertar o cuidado ao indivíduo; com o telemonitoramento tem-se realizado uma assistência de enfermagem em Estomaterapia sistematizada, qualificada, abrangente e diferenciada.

Para o futuro, espera-se que seja possível despertar em outros estudantes o interesse em participar deste projeto, ampliando o conhecimento e atividades a serem realizadas. 
Que se alcance o quantitativo de todos os usuários atendidos na clínica para melhor resultado de suas condições de saúde. Mais pesquisas realizadas pelos estudantes sob orientação dos docentes e enfermeiros, para que haja exploração dos conhecimentos.

\section{Referências}

1. SANTOS, Déborah Machado dos. Construção e validação de instrumentos para a sistematização da assistência de enfermagem em uma clínica de enfermagem em estomaterapia. 2018. 303 f. Tese (Doutorado em Enfermagem) - Faculdade de Enfermagem, Universidade do Estado do Rio de Janeiro, Rio de Janeiro, 2018.

2. CESARETTI, Isabel Umbelina Ribeiro; DIAS, Sonia Maria. Estomaterapia: uma especialidade em evolução. Acta Paul. Enf.; v. 4, n. 15, p. 79-86, out./dez. 2002.

3. BRASIL. Instituto Nacional de Câncer. Coordenação de Prevenção e Vigilância. Estimativa 2018: incidência de câncer no Brasil / Instituto Nacional de Câncer. Coordenação de Prevenção e Vigilância. - Rio de Janeiro: INCA, 2017.

4. SILVA, Lígia.; LOPES, Maria Helena Baena de Moraes. Incontinência urinária em muIheres: razões da não procura por tratamento. Rev. Esc. Enferm. USP, São Paulo, v. 1, n. 43, p.72-8, mar. 2009.

5. BLAKE, Holly. Innovation in practice: mobile phone technology in patient care. $\mathbf{B r} \mathbf{J}$ CommunityNurs., v. 13, n. 4, p.160-2, Apr. 2008.

6. DELPHINO, Tallita Mello. Efeito do acompanhamento por telefone na recuperação cirúrgica de idosos submetidos à cirurgia de facectomia: estudo clínico randomizado. [Dissertação de Mestrado em Enfermagem]. Faculdade de Enfermagem da Universidade Federal Fluminense, Rio de Janeiro, 2016. 